\title{
Advances in target therapy in lung cancer
}

\author{
Jean-Paul Sculier, Thierry Berghmans and Anne-Pascale Meert
}

Affiliation: Service des Soins Intensifs et Urgences Oncologiques \& Oncologie Thoracique, Institut Jules Bordet, Centre des Tumeurs de l'Université Libre de Bruxelles (ULB), Brussels, Belgium.

Correspondence: Jean-Paul Sculier, Service des Soins Intensifs et Urgences Oncologiques \& Oncologie Thoracique, Institut Jules Bordet, 1 Rue Héger-Bordet, B-1050 Brussels, Belgium. E-mail: sculierabordet.be

ABSTRACT Herein, we have reviewed and analysed recent literature, published in 2013 and early 2014, in the context of pre-existing data. Considered target therapies were tyrosine kinase inhibitors of active epidermal growth factor receptor mutations (e.g. erlotinib, gefinitib and afatinib), anaplastic lymphoma kinase rearrangements (e.g. crizotinib) or angiogenesis (drugs under development), or monoclonal antibodies against vascular endothelial growth factor (e.g. bevacizumab) or epidermal growth factor receptors (e.g. cetuximab). The therapeutic project has to consider tyrosine kinase inhibitors in the case of nonsmall cell lung cancer with active epidermal growth factor receptor mutations or anaplastic lymphoma kinase rearrangement. However, these drugs should not be used in the absence of the targeted genetic abnormalities.

O @ERSpublications

Targeted therapies are modifying the therapeutic approach in NSCLC with specific oncogenic mutations http://ow.ly/HeVMZ

\section{Introduction}

At present, target therapy is part of the routine management for patients with advanced nonsmall cell lung cancer (NSCLC). This article is based on the systematic review performed for the 2014 update of the European Lung Cancer Working Party guidelines [1], and will focus on the most recently published randomised clinical trials (RCTs), systematic reviews and meta-analyses. The vast majority of published RCTs have been performed by the pharmaceutical industry for registration purposes.

Target therapy consists of tyrosine kinase inhibitors (TKI) of active epidermal growth factor receptor (EGFR) mutations (e.g. erlotinib, gefinitib and afatinib), anaplastic lymphoma kinase (ALK) rearrangement (e.g. crizotinib) or angiogenesis (drugs under development), or monoclonal antibodies against vascular endothelial growth factor (VEGF) (e.g. bevacizumab) or EGFRs (e.g. cetuximab). In 2014, the European Medicines Agency (EMA) approved five of these drugs for the treatment of advanced NSCLC: erlotinib, gefinitib, afatinib, crizotinib and bevacizumab.

\section{TKIs for actively mutated EGFRs}

Due to the number of articles published on this topic in 2013 and 2014, this will be the main section of the review. From a historical point of view, two TKIs (erlotinib [2] and gefitinib [3]) were initially assessed in comparison to best supportive care as salvage therapy in advanced NSCLC in unselected patient populations. There was a small, but statistically significant, survival advantage in one of the RCTs (table 1). Some clinical characteristics were found to be associated with a response to EGFR-TKI: female sex, adenocarcinoma, being a nonsmoker and East Asian race. Academic research rapidly identified the target of these drugs, an abnormal EGFR [4]. Active mutations, by amino acid substitution or deletion on some exons (mainly 19 and 21), leads to permanent activation of the tyrosine kinase of the receptor. The autophosphorylated EGFR activates downstream effectors, such as mitogen-activated protein kinase, STAT3 or AKT, via proliferative and cell survival signals. The search for these mutations has rapidly

Received: Nov 172014 | Accepted after revision: Dec 112014

Conflict of interest: None declared.

Provenance: Submitted article, peer reviewed.

Copyright CERS 2015. ERR articles are open access and distributed under the terms of the Creative Commons Attribution Non-Commercial Licence 4.0. 


\begin{tabular}{|c|c|c|c|c|c|c|}
\hline $\begin{array}{l}\text { First author } \\
\text { [ref.] }\end{array}$ & $\begin{array}{l}\text { Treatment } \\
\text { arm }\end{array}$ & $\begin{array}{c}\text { Patients } \\
\mathrm{n}\end{array}$ & $\begin{array}{c}\text { Objective } \\
\text { response \% }\end{array}$ & p-value & $\begin{array}{l}\text { Median survival } \\
\text { months }\end{array}$ & p-value \\
\hline \multirow[t]{2}{*}{ SHEPHERD [2] } & Erlotinib & 488 & 8.9 & $<0.001$ & 6.7 & $<0.001$ \\
\hline & Placebo & 243 & 1 & & 4.7 & \\
\hline \multirow[t]{2}{*}{ THATCHER [3] } & Gefitinib & 1129 & 8 & $<0.0001$ & 5.6 & 0.09 \\
\hline & Placebo & 563 & 1.3 & & 5.1 & \\
\hline
\end{tabular}

developed and is an important test to be performed in the routine management of advanced NSCLC. In 2011, a joint statement of the International Association for the Study of Lung Cancer/American Thoracic Society/European Respiratory Society strongly recommended the routine determination of molecular and genetic markers in adenocarcinoma [5]. This statement is now part of the definition of these tumours.

Recent publications have enabled the indications of TKIs in advanced NSCLC to be better defined, thus it is important to consider the three types of tumours separately: wild-type EGFR mutations, active EGFR mutations and unselected populations.

NSCLC with wild-type EGFR: no role for TKI

The academic trial TAILOR, which was published in 2013 [6], assessed the role of the TKI erlotinib in comparison to chemotherapy (docetaxel) as second-line therapy for advanced NSCLC with wild-type EGFR. The primary end-point was survival. According to the criteria of patient eligibility in the study, EGFR had to be genotyped for mutational status of exons 19-21 in two independent laboratories using two different techniques (Sanger sequencing and restriction fragment length polymorphism). Of 702 registered patients, 540 were genotyped and 222 were enrolled. The results are summarised in table 2. Progression-free survival was significantly better with docetaxel than erlotinib (adjusted HR 0.71, p=0.02) and there was an improvement in overall survival in favour of docetaxel (adjusted HR 0.73, p=0.05). Recently, a similar Chinese trial compared pemetrexed to gefitinib in wild-type EGFR nonsquamous NSCLC patients [7]. In total, 157 assessable patients were enrolled (table 2). Progression-free survival was significantly better with pemetrexed than gefitinib (HR 0.54, $\mathrm{p}<0.001)$ and there was a trend for an improvement in overall survival in favour of docetaxel (adjusted HR 0.72, $p=0.077$ ). In terms of evidence-based medicine, these trials are strongly against the use of TKIs in non-mutated EGFR NSCLC.

A meta-analysis of the literature has been carried out with a RCT comparing EGFR-TKI monotherapy or combination EGFR-TKI chemotherapy with chemotherapy or placebo [8]. Treatment efficacy was assessed by separating the EGFR-TKIs for the EGFR mutation-positive and EGFR mutation-negative subgroups of each trial. The presence of a mutation was not a criterion for patient selection and, thus, post hoc analysis only has an exploratory value. Within the 23 eligible trials (first-line therapy: $n=13$; second-line therapy: $n=7$; maintenance therapy: $n=3$ ), a total of 14570 patients were randomised and EGFR mutation status was known in $31 \%$ of patients. In non-mutated EGFR patients, the hazard ratio was $1.06(\mathrm{p}=0.35)$ for first-line therapy, $1.23(\mathrm{p}=0.01)$ for second-line therapy and $0.81(\mathrm{p}=0.02)$ for maintenance therapy. TKI treatment had no impact on overall survival. These data do not support the use of these drugs in wild-type EGFR tumours.

NSCLC with active EGFR mutations: TKI strongly indicated

Six randomised phase III trials have compared TKIs to chemotherapy as first-line treatment for sensitive EGFR-mutated lung cancer (table 3), and all show significantly better response rates and progression free-survival

TABLE 2 Randomised phase III trials in wild-type epidermal growth factor receptor lung cancer: salvage therapy

\begin{tabular}{lcccccc}
$\begin{array}{l}\text { First author } \\
\text { [ref.] }\end{array}$ & $\begin{array}{c}\text { Treatment } \\
\text { arm }\end{array}$ & $\begin{array}{c}\text { Patients } \\
\mathbf{n}\end{array}$ & $\begin{array}{c}\text { 0bjective } \\
\text { response \% }\end{array}$ & p-value & $\begin{array}{c}\text { Median survival } \\
\text { months }\end{array}$ & p-value \\
\hline GARASSINo [6] & Erlotinib & 112 & 3 & 0.001 & 5.4 & 0.05 \\
\multirow{2}{*}{ ZHou [7] } & Docetaxel & 110 & 15.5 & & 8.2 & 0.077 \\
& Gefitinib & 81 & 12.3 & NS & 9.6 & 12.4 \\
\hline
\end{tabular}

NS: nonsignificant. 
but no difference in overall survival. This is explained by a crossover between both non-cross resistant therapies at relapse or failure. Three studies have recently been published, two in 2013 and one in 2014.

The first study was conducted by the North-East Japan Study Group [10], and is an update of a prior preliminary publication for overall survival [9]. The study compared gefitinib with carboplatin and paclitaxel in 228 randomised patients. The median survival time was 27.7 months for the TKI group and 26.6 months for the chemotherapy group (HR 0.887, p=0.483). Post-protocol administered chemotherapy was analysed. The survival of the 186 patients who received platinum throughout their treatment was not statistically different from that of the 40 patients who never received platinum. In the gefitinib group, 82 (72\%) patients received at least one subsequent regimen. Among these, 74 (65\%) patients were treated with a platinum doublet regimen. For those who received initial chemotherapy, 109 (95.6\%) patients received a TKI as second-line treatment.

The second study, LUX-Lung 3 [14], compared afatinib to chemotherapy (cisplatin plus pemetrexed). A total of 1269 patients were screened, of these 345 with a positive mutation for EGFR, mainly from East Asia, were randomised. Progression-free survival was significantly improved with afatinib (HR 0.58, p<0.001).

The third study was performed in China and also compared afatinib to cisplatin-based chemotherapy with gemcitabine [15]. Similar results were obtained with significantly longer progression-free survival for the TKIs (HR 0.28, $\mathrm{p}<0.0001$ ).

In the previously mentioned meta-analysis [8], progression-free survival was significantly better for TKIs as first-line therapy (HR 0.43, p<0.001), second-line therapy (HR 0.34, p<0.001) and maintenance therapy (HR $0.15, \mathrm{p}<0.001$ ) in the EGFR mutation-positive subgroup. EGFR-TKI treatment had no impact on overall survival, confirming the results of the various RCTs. Another meta-analysis was performed to compare gefitinib, erlotinib, afatinib and chemotherapy as first-line therapies for patients with advanced NSCLC harbouring EGFR-activating mutations [16]. Indeed there is no RCT comparing different TKIs in that indication. The authors confirmed that gefitinib, erlotinib and afatinib out-performed chemotherapy in terms of progression-free survival, overall response rate and disease control rate, but not overall survival. The authors made indirect comparisons between the various TKIs and found no statistically significant differences among them.

An important question is which type of treatment should be proposed when disease progresses during TKI treatment [17]. There is a lack of studies with high levels of evidence. Various approaches may be possible, such as examining for small cell lung cancer (this requires re-biopsy) or considering platinum-based

TABLE 3 Randomised phase III trials comparing tyrosine kinase inhibitors versus chemotherapy as first-line treatment for sensitive epidermal growth factor receptor-mutated lung cancer

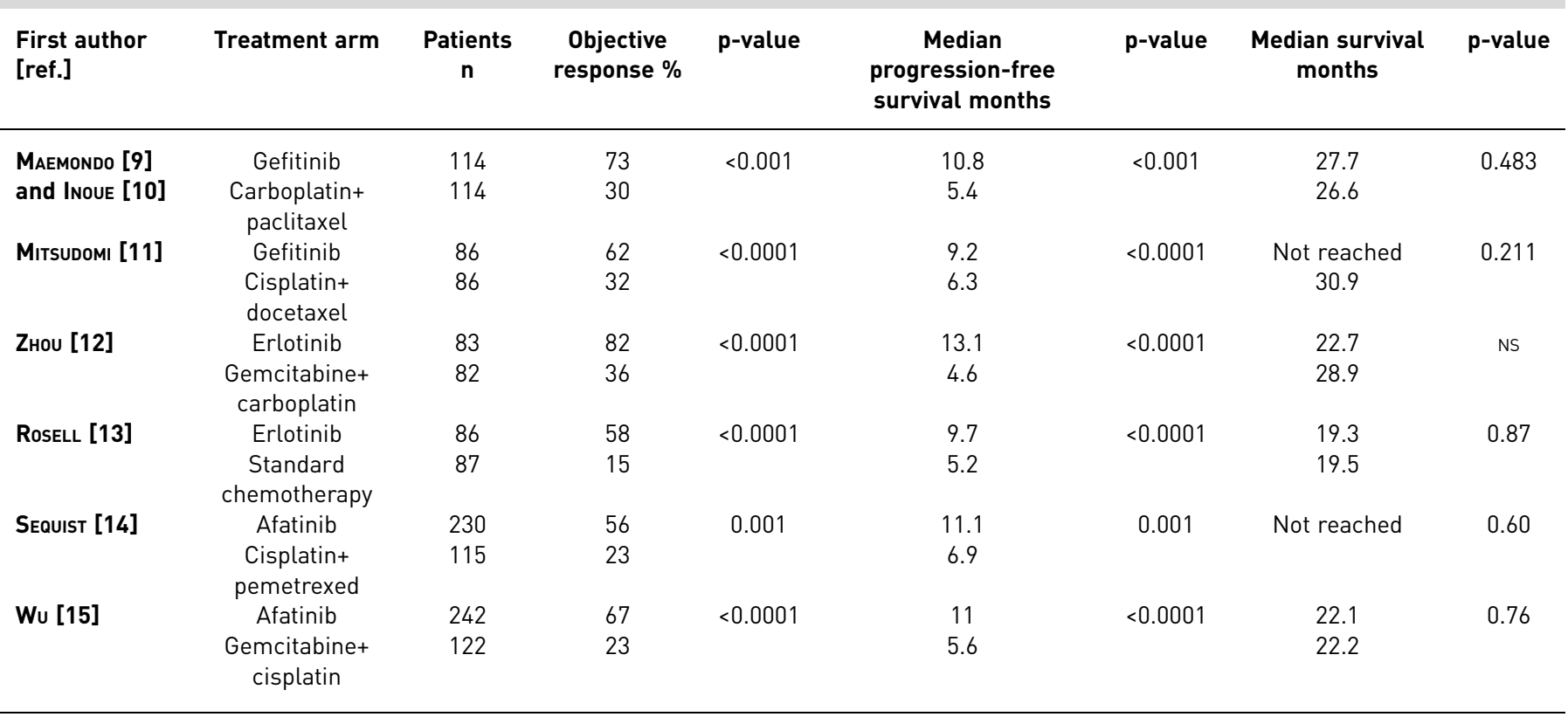

NS: nonsignificant. 
chemotherapy if the patient is chemonaive and then retreatment by TKI if there is further failure of chemotherapy. Other options include continuation of TKIs in case of slow growing tumours, changing to another TKI, local treatment (as in the management of oligometastases), or the addition of a cytotoxic drug. To understand the mechanism of resistance (resistant mutations such as T790M, MET amplification, etc.), re-biopsy can be performed and clinical trials considered if available.

\section{Unselected populations: not very useful information}

A lot of trials are available for those populations for whom EGFR genotyping has not been performed and are usually enriched by patients with clinical characteristics predicting a better chance to respond to TKI, such as race, sex, histology or smoking status. Various approaches have been tested. For first-line treatment, TKIs have been assessed in combination with chemotherapy, in comparison to chemotherapy, as sequential therapy versus best supportive care for patients not eligible for chemotherapy, or for maintenance therapy. As salvage therapy, they have been compared to best supportive care or chemotherapy or tested after the first TKI failed. Finally, some trials have tested intercalated TKIs with chemotherapy.

Recently published RCTs in unselected populations have dealt with salvage therapy [18, 19] and intercalation [20-22]. A study from Greece has compared pemetrexed with erlotinib as second- or third-line therapy [18]. Of the 357 patients enrolled between January 2006 and April 2010, 332 patients (166 per arm) were evaluable for response and toxicity. Partial response was documented in 10 (11.4\%) patients in the pemetrexed arm and 15 (9\%) patients in the erlotinib arm. The median progression-free survival was 2.9 and 3.6 months for the pemetrexed and erlotinib arms, respectively, and median overall survival was 10.1 and 8.2 months, respectively. None of these differences were statistically significant. EGFR mutation status was assessed in 123 patients (62 in the pemetrexed arm and 61 in the erlotinib arm) who had adequate tumour tissue available. EGFR mutations were detected in five and six patients, respectively.

A Japanese trial also compared erlotinib and docetaxel in an unselected population [19]. Respectively, 150 and 151 patients were randomised from August 2009 to July 2012. Median progression-free survival for erlotinib and docetaxel was 2.0 and 3.2 months, respectively, and median overall survival was 14.8 and 12.2 months, respectively. None of these differences were statistically different. EGFR wild-type NSCLC was present in 109 and 90 patients in the erlotinib and docetaxel groups, respectively. In the subset analysis of EGFR wild-type tumours, median progression-free survival for erlotinib and docetaxel was 1.3 and 2.9 months (HR 1.45, $\mathrm{p}=0.01$ ), respectively, and median overall survival was 9.0 and 10.1 months (HR $0.98, p=0.91)$, respectively. The results of the Japanese trial are in agreement with the data presented in the previous section on NSCLC with wild-type EGFR.

In patients with no prior treatment, intercalated combination of chemotherapy and erlotinib has been the topic of a phase III double-blind RCT, FASTACT-2 [20]. Asian patients were randomised to receive six cycles of chemotherapy with gemcitabine $\left(1250 \mathrm{mg} \cdot \mathrm{m}^{-2}\right.$ on days 1 and 8$)$ plus platinum (carboplatin $5 \times$ area under the curve or cisplatin $75 \mathrm{mg} \cdot \mathrm{m}^{-2}$ on day 1) with intercalated erlotinib (150 mg.day ${ }^{-1}$ orally on days $\left.15-28\right)$, or chemotherapy plus oral placebo. From April 2009 to September 2010, a total of 451 patients were randomly assigned to chemotherapy plus erlotinib $(n=226)$ or chemotherapy plus placebo $(n=225)$. Tumour samples were available for EGFR mutation analysis from 301 patients and could be analysed in 283 patients. EGFR mutation status was determined for 241 (53\%) patients: 136 (56\%) patients had EGFR wild-type status, eight (3\%) patients had single resistance mutations and 97 (40\%) patients had EGFR-activating mutations. 210 had unknown EGFR mutation status. Progression-free survival was significantly prolonged with chemotherapy plus erlotinib (HR 0.57, $\mathrm{p}<0.0001$ ). Median overall survival for patients in the chemotherapy plus erlotinib and chemotherapy plus placebo groups was 18.3 and 15.2 months (HR $0.79, p=0.042$ ), respectively. Nevertheless, a treatment benefit was only observed in patients with an activating EGFR gene mutation both for progression-free survival (HR 0.25, $\mathrm{p}<0.0001$ ) and overall survival (HR 0.48, $\mathrm{p}=0.0092$ ).

Intercalation has also been the subject of two phase II RCTs as salvage therapy. The first RCT compared pemetrexed and erlotinib to either pemetrexed or erlotinib alone as second-line treatment for 240 never-smokers with nonsquamous NSCLC [21]. Pemetrexed plus erlotinib significantly improved progression-free survival. EGFR genotyping was possible in 43 patients with 24 having sensitising mutations. The second RCT, the NVALT-10 study which was conducted in the Netherlands, randomised patients between erlotinib in monotherapy or erlotinib for 15 days intercalated with four 21-day cycles of docetaxel for squamous cell histology or pemetrexed for nonsquamous cell histology [22]. A total of 231 patients were eligible. In 60 patients, EGFR mutation was determined with three positive mutations. Progression-free survival (HR 0.7, p=0.06) and overall survival (HR 0.67, p=0.01) were in favour of the combination arm.

For maintenance therapy, a Chinese meta-analysis examined published data of RCTs where EGFR TKIs were compared against placebo in maintenance regimens [23]. It included six trials with 3758 patients. 
Maintenance therapy improved objective response rate $(\mathrm{OR} 1.29, \mathrm{p}<0.01)$ and progression-free survival (HR 0.77, $\mathrm{p}<0.01$ ) but was unable to prolong overall survival ( $\mathrm{HR} 0.94, \mathrm{p}=0.10$ ). The heterogeneity in terms of patients with good clinical characteristics and the lack of EGFR genotyping were major limitations of the meta-analysis.

Two studies merit special attention. The first, the TOPICAL study, was a double-blind, placebo-controlled, phase III RCT that was performed at 78 centres in the UK [24]. It was performed in patients who were unsuitable for chemotherapy because of poor Eastern Cooperative Oncology Group performance status $(\geqslant 2)$, the presence of several comorbidities or both. They were randomly assigned to receive oral placebo or erlotinib until disease progression or unacceptable toxicity. Between April 2005 and April 2009, 350 and 320 patients were randomly assigned to receive erlotinib or placebo, respectively. Overall survival did not differ between arms (HR 0.94, p=0.46). DNA was available for 390 patients (58\% of total study population). Occurrence of the activating EGFR mutation in the study population was only $7 \%$ (28 out of 390 ).

The second study, LUX-Lung 1, assessed afatinib, an irreversible ErbB-family blocker, in patients with advanced lung adenocarcinoma with previous treatment failure on EGFR TKI (erlotinib or gefitinib) [25]. Between May 2008 and September 2009, 585 patients were randomly allocated to afatinib $(n=390)$ or to placebo ( $\mathrm{n}=195)$. Median overall survival was 10.8 and 12.0 months (HR 1.08, p=0.74), respectively. For the 96 patients who had EGFR mutation-positive tumour tissue, progression-free survival was longer for those treated with afatinib (HR 0.51, $\mathrm{p}=0.009$ ). By contrast, there was no difference in progression-free survival between treatment groups for the 45 patients who were EGFR mutation-negative (HR 0.61, $\mathrm{p}=0.22$ ).

In 2014 in the European Union, EGFR TKIs have only received EMA registration for NSCLC with active mutation, except erlotinib which can be administered for maintenance therapy or as salvage therapy following platinum-based chemotherapy failure. The data we have reviewed does not support the administration of any TKI without genotyping EGFR. Unfortunately, in some cases, tissue cannot be obtained and thus the test cannot be performed. In those situations, we suggest using the nomogram developed by GIRARD et al. [26]. It allows the presence of EGFR mutations in lung adenocarcinoma in non-Asian patients to be predicted when mutational profiling is not available or possible. It is based on simple clinical characteristics: age, sex, tobacco quantity smoked (in pack-years), time since quitting smoking (years), predominant histological subtype and cancer stage. It estimates the probability of the presence of an EGFR activating mutation.

\section{TKIs for tumours with ALK rearrangement}

Chromosomal rearrangements of the ALK gene have been associated with marked clinical responses to crizotinib, an oral TKI targeting ALK, as shown in a prior expanded cohort following a phase I trial [27]. These data prompted phase III, open-label trials comparing crizotinib with chemotherapy (table 4). As salvage therapy [28], a total of 347 patients with locally advanced or metastatic ALK-positive lung cancer who had received one prior platinum-based regimen were randomly assigned to receive oral treatment with crizotinib (250 mg twice daily) or chemotherapy with pemetrexed or docetaxel every 3 weeks. Progression-free survival (HR 0.49, $\mathrm{p}<0.001$ ) and response rates $(65 \%$ versus $20 \%, \mathrm{p}<0.001$ ) were both in favour of crizotinib. A preliminary analysis of overall survival showed no significant improvement with crizotinib compared with chemotherapy (HR 1.02, p=0.54). As first-line therapy [29], crizotinib was compared to chemotherapy using pemetrexed plus cisplatin or carboplatin in nonsquamous NSCLC. A total of 343 patients were randomised. Progression-free survival (HR 0.45, p<0.001) and response rates

TABLE 4 Randomised phase III trials in anaplastic lymphoma kinase-positive lung cancer

Treatment arm Patients Objective p-value Median progression-free

n responses $\%$ survival months

p-value Median survival p-value

n

\begin{tabular}{|c|c|c|c|c|c|c|c|c|}
\hline \multicolumn{9}{|l|}{ Salvage therapy } \\
\hline \multirow[t]{2}{*}{ SHAW [28] } & Crizotinib & 173 & 65 & $<0.001$ & 7.7 & $<0.001$ & 20.3 & NS \\
\hline & $\begin{array}{l}\text { Docetaxel or } \\
\text { pemetrexed }\end{array}$ & 174 & 20 & & 3.0 & & 22.8 & \\
\hline \multicolumn{9}{|l|}{ First-line therapy } \\
\hline \multirow[t]{2}{*}{ SoLomon [29] } & Crizotinib & 172 & 74 & $<0.001$ & 10.9 & $<0.001$ & Not reached & NS \\
\hline & $\begin{array}{l}\text { Pemetrexed plus } \\
\text { cisplatin or } \\
\text { carboplatin }\end{array}$ & 171 & 45 & & 7.0 & & Not reached & \\
\hline
\end{tabular}

NS: nonsignificant. 
( $74 \%$ versus $45 \%, \mathrm{p}<0.001$ ) were also both in favour of crizotinib. Crizotinib has also been shown to have marked antitumor activity in patients with advanced ROS1-rearranged NSCLC [30].

\section{Antiangiogenic TKIs}

A meta-analysis has been performed on the published RCTs comparing chemotherapy plus multi-targeted antiangiogenic TKIs to chemotherapy alone in advanced NSCLC [31]. The drugs considered were sorafenib, sunitinib, cediranib, vandetanib, BIBF 1120, pazopanib and axitinib. Six RCTs involving 3337 patients were analysed. Compared to chemotherapy alone, chemotherapy plus multi-targeted antiangiogenic TKIs significantly increased the response rate and progression-free survival, but not overall survival. At present, antiangiogenic drugs are not registered by the EMA for the management of lung cancer.

\section{Anti-VEGF monoclonal antibodies}

Bevacizumab is registered by the EMA as an antiangiogenic drug for the treatment of lung cancer. A systematic review and meta-analysis of randomised, phase II/III trials adding bevacizumab to platinum-based chemotherapy as first-line treatment in advanced NSCLC has been performed [32]. Data from 2194 patients (1313 bevacizumab, 881 controls) from four phase II and III trials were analysed. Compared with chemotherapy alone, bevacizumab significantly prolonged overall survival (HR 0.90, $\mathrm{p}=0.03$ ) and progression-free survival (HR 0.72, $\mathrm{p}<0.001$ ). It also significantly increased the risk of high-grade proteinuria, hypertension, haemorrhagic events, neutropenia and febrile neutropenia.

\section{Anti-EGFR monoclonal antibodies}

Contrary to the Food and Drug Administration, cetuximab, a monoclonal antibody targeting EGFR, has not been accepted by the EMA. A meta-analysis of individual patient data from randomised trials has assessed the addition of cetuximab to chemotherapy as first-line treatment for advanced NSCLC [33]. It included individual patient efficacy data from 2018 patients from four RCTs. The addition of cetuximab to chemotherapy significantly improved overall survival (HR 0.88, median 10.3 versus 9.4 months; $\mathrm{p}=0.009$ ), progression-free survival (HR, 0.90 , median 4.7 versus 4.5 months; $\mathrm{p}=0.045)$ and response (OR 1.46, 32.2\% versus $24.4 \%$; $\mathrm{p}<0.001)$ compared with chemotherapy alone. The authors concluded a favourable benefit/ risk ratio.

\section{Conclusion}

At present, evidence supports the fact that the therapeutic project has to consider TKIs in the case of NSCLC with active EGFR mutations or ALK rearrangement. When these genetic abnormalities are not present on the tumour, these drugs should not be prescribed.

\section{References}

1 Berghmans T, Cortot A, CsToth I, et al. Les recommandations de pratique clinique de l'European Lung Cancer Working Party: Mises à jour. [Guidelines of clinical practice made by the European Lung Cancer Working Party: updates]. Rev Med Brux 2014; 35: 132-133.

2 Shepherd FA, Rodrigues Pereira J, Ciuleanu T, et al. Erlotinib in previously treated non-small-cell lung cancer. N Engl J Med 2005; 353: 123-132.

3 Thatcher N, Chang A, Parikh P, et al. Gefitinib plus best supportive care in previously treated patients with refractory advanced non-small-cell lung cancer: results from a randomised, placebo-controlled, multicentre study (Iressa Survival Evaluation in Lung Cancer). Lancet 2005; 366: 1527-1537.

4 Lynch TJ, Bell DW, Sordella R, et al. Activating mutations in the epidermal growth factor receptor underlying responsiveness of non-small-cell lung cancer to gefitinib. N Engl J Med 2004; 350: 2129-2139.

5 Travis WD, Brambilla E, Noguchi M, et al. International Association for the Study of Lung Cancer/American Thoracic Society/European Respiratory Society international multidisciplinary classification of lung adenocarcinoma. J Thorac Oncol 2011; 6: 244-285.

6 Garassino MC, Martelli O, Broggini M, et al. Erlotinib versus docetaxel as second-line treatment of patients with advanced non-small-cell lung cancer and wild-type EGFR tumours (TAILOR): a randomised controlled trial. Lancet Oncol 2013; 14: 981-988.

7 Zhou Q, Cheng Y, Yang JJ, et al. Pemetrexed versus gefitinib as a second-line treatment in advanced nonsquamous nonsmall-cell lung cancer patients harboring wild-type EGFR (CTONG0806): a multicenter randomized trial. Ann Oncol 2014; 25: 2385-2391.

8 Lee CK, Brown C, Gralla RJ, et al. Impact of EGFR inhibitor in non-small cell lung cancer on progression-free and overall survival: a meta-analysis. J Natl Cancer Inst 2013; 105: 595-605.

9 Maemondo $\mathrm{M}$, Inoue $\mathrm{A}$, Kobayashi $\mathrm{K}$, et al. Gefitinib or chemotherapy for non-small-cell lung cancer with mutated EGFR. N Engl J Med 2010; 362: 2380-2388.

10 Inoue A, Kobayashi K, Maemondo M, et al. Updated overall survival results from a randomized phase III trial comparing gefitinib with carboplatin-paclitaxel for chemo-naive non-small cell lung cancer with sensitive EGFR gene mutations (NEJ002). Ann Oncol 2013; 24: 54-59.

11 Mitsudomi T, Morita S, Yatabe Y, et al. Gefitinib versus cisplatin plus docetaxel in patients with non-small-cell lung cancer harbouring mutations of the epidermal growth factor receptor (WJTOG3405): an open label, randomised phase 3 trial. Lancet Oncol 2010; 11: 121-128. 
12 Zhou $\mathrm{C}, \mathrm{Wu} \mathrm{YL}$, Chen G, et al. Erlotinib versus chemotherapy as first-line treatment for patients with advanced EGFR mutation-positive non-small-cell lung cancer (OPTIMAL, CTONG-0802): a multicentre, open-label, randomised, phase 3 study. Lancet Oncol 2011; 12: 735-742.

13 Rosell R, Carcereny E, Gervais R, et al. Erlotinib versus standard chemotherapy as first-line treatment for European patients with advanced EGFR mutation-positive non-small-cell lung cancer (EURTAC): a multicentre, open-label, randomised phase 3 trial. Lancet Oncol 2012; 13: 239-246.

14 Sequist LV, Yang JC, Yamamoto N, et al. Phase III study of afatinib or cisplatin plus pemetrexed in patients with metastatic lung adenocarcinoma with EGFR mutations. J Clin Oncol 2013; 31: 3327-3334.

$15 \mathrm{Wu} \mathrm{YL}, \mathrm{Lu} \mathrm{S}$, Cheng Y, et al. Efficacy and safety of pemetrexed/cisplatin versus gemcitabine/cisplatin as first-line treatment in Chinese patients with advanced nonsquamous non-small cell lung cancer. Lung Cancer 2014; 85: 401-407.

16 Haaland B, Tan PS, de Castro G Jr, et al. Meta-analysis of first-line therapies in advanced non-small-cell lung cancer harboring EGFR-activating mutations. J Thorac Oncol 2014; 9: 805-811.

17 Chong CR, Janne PA. The quest to overcome resistance to EGFR-targeted therapies in cancer. Nat Med 2013; 19: 1389-1400.

18 Karampeazis A, Voutsina A, Souglakos J, et al. Pemetrexed versus erlotinib in pretreated patients with advanced non-small cell lung cancer: a Hellenic Oncology Research Group (HORG) randomized phase 3 study. Cancer 2013; 119: 2754-2764.

19 Kawaguchi T, Ando M, Asami K, et al. Randomized phase III trial of erlotinib versus docetaxel as second- or third-line therapy in patients with advanced non-small-cell lung cancer: Docetaxel and Erlotinib Lung Cancer Trial (DELTA). J Clin Oncol 2014; 32: 1902-1908.

$20 \mathrm{Wu}$ YL, Lee JS, Thongprasert S, et al. Intercalated combination of chemotherapy and erlotinib for patients with advanced stage non-small-cell lung cancer (FASTACT-2): a randomised, double-blind trial. Lancet Oncol 2013; 14: 777-786.

21 Lee DH, Lee JS, Kim SW, et al. Three-arm randomised controlled phase 2 study comparing pemetrexed and erlotinib to either pemetrexed or erlotinib alone as second-line treatment for never-smokers with non-squamous non-small cell lung cancer. Eur J Cancer 2013; 49: 3111-3121.

22 Aerts JG, Codrington H, Lankheet NA, et al. A randomized phase II study comparing erlotinib versus erlotinib with alternating chemotherapy in relapsed non-small-cell lung cancer patients: the NVALT-10 study. Ann Oncol 2013; 24: 2860-2865.

23 Alimujiang S, Zhang T, Han ZG, et al. Epidermal growth factor receptor tyrosine kinase inhibitor versus placebo as maintenance therapy for advanced non- small-cell lung cancer: a meta-analysis of randomized controlled trials. Asian Pac J Cancer Prev 2013; 14: 2413-2419.

24 Lee SM, Khan I, Upadhyay S, et al. First-line erlotinib in patients with advanced non-small-cell lung cancer unsuitable for chemotherapy (TOPICAL): a double-blind, placebo-controlled, phase 3 trial. Lancet Oncol 2012; 13: $1161-1170$

25 Miller VA, Hirsh V, Cadranel J, et al. Afatinib versus placebo for patients with advanced, metastatic non-small-cell lung cancer after failure of erlotinib, gefitinib, or both, and one or two lines of chemotherapy (LUX-Lung 1): a phase $2 \mathrm{~b} / 3$ randomised trial. Lancet Oncol 2012; 13: 528-538.

26 Girard N, Sima CS, Jackman DM, et al. Nomogram to predict the presence of EGFR activating mutation in lung adenocarcinoma. Eur Respir J 2012; 39: 366-372.

27 Kwak EL, Bang YJ, Camidge DR, et al. Anaplastic lymphoma kinase inhibition in non-small-cell lung cancer. N Engl J Med 2010; 363: 1693-1703.

28 Shaw AT, Kim DW, Nakagawa K, et al. Crizotinib versus chemotherapy in advanced ALK-positive lung cancer. N Engl J Med 2013; 368: 2385-2394.

29 Solomon BJ, Mok T, Kim DW, et al. First-line crizotinib versus chemotherapy in ALK-positive lung cancer. N Engl J Med 2014; 371: 2167-2177.

30 Shaw AT, Ou SH, Bang YJ, et al. Crizotinib in ROS1-rearranged non-small-cell lung cancer. N Engl J Med 2014; 371: 1963-1971.

31 Li J, Huang S, Zheng W, et al. The role of vascular epithelial growth factor receptor-tyrosine kinase inhibitors in the treatment of advanced breast cancer: a meta-analysis of 12 randomized controlled trials. Curr Med Res Opin 2013; 29: 1691-1699.

32 Soria JC, Mauguen A, Reck M, et al. Systematic review and meta-analysis of randomised, phase II/III trials adding bevacizumab to platinum-based chemotherapy as first-line treatment in patients with advanced non-small-cell lung cancer. Ann Oncol 2013; 24: 20-30.

33 Pujol JL, Pirker R, Lynch TJ, et al. Meta-analysis of individual patient data from randomized trials of chemotherapy plus cetuximab as first-line treatment for advanced non-small cell lung cancer. Lung Cancer 2014; 83: 211-218. 